\title{
Numerical Computation of Electric and Magnetic Fields
}

Second Edition 


\section{Join Us on the Internet}

WWW: hHtp://www.thomson.com

EMAlL: findi@@kiosk.thomson.com

thomson.com is the on-line portal for the products, services and resources available from International Thomson Publishing (ITP).

This Internet kiosk gives users immediate access to more than 34 ITP publishers and over 20,000 products. Through thomson.com Internet users can search catalogs, examine subject-specific resource centers and subscribe to electronic discussion lists. You can purchase ITP products from your local bookseller, or directly through thomson.com.

Visit Chapman \& Hall's Internet Resource Center for information on our new publications, links to useful sites on the World Wide Web and an opportunity to join our e-mail mailing list. Point your browser to: http://www.chaphall.com/chaphall.html

or http://www.chaphall.com/chaphall/electeng.html for Electrical Engineering

A service of $\mathbf{I} \mathbf{P}^{\circledR}$ 


\section{Numerical Computation of Electric and Magnetic Fields}

Charles W. Steele 
Cover design: Curtis Tow Graphics

Copyright $@ 1997$ by Springer Science+Business Media Dordrecht

Originally published by Chapman \& Hall in 1997

Softcover reprint of the hardcover 2nd edition 1997

All rights reserved. No part of this book covered by the copyright hereon may be reproduced or used in any form or by any means-graphic, electronic, or mechanical, including photocopying, recording, taping, or information storage and retrieval systems-without the written permission of the publisher.

\section{$\begin{array}{lllllllllllllllll}1 & 2 & 3 & 5 & 6 & 7 & 8 & 9 & 10 & \text { XXX } 010099 & 9897\end{array}$}

\section{Library of Congress Cataloging-in-Publication Data}

\section{Steele, Charles W.}

Numerical computation of electric and magnetic fields / Charles W. Steele. - 2nd ed.

$$
\text { p. } \mathrm{cm} \text {. }
$$

Includes bibliographical references and index.

ISBN 978-1-4613-7766-5 ISBN 978-1-4615-6035-7 (eBook)

DOI 10.1007/978-1-4615-6035-7

1. Electric fields--Measurement. 2. Magnetic fields--Measurement.

3. Numerical analysis. I. Title.

QC665.E38S74 1996

530.1'41'015194--dc20

96-17140

\section{British Library Cataloguing in Publication Data available}


To

CANDACE 


\section{CONTENTS}

Dedication

Page

Preface

Preface to First Edition xiii

1. Introduction 1

2. Field Properties 3

2.1 Introduction 3

2.2 Maxwell's Equations in the Dynamic, Quasi-Static, and Static Cases

2.3 Polarization and Magnetization 6

2.4 Laws for Static Fields in Unbounded Regions 9

2.5 Integral Representations for Quasi-Static Fields Using the $\begin{array}{ll}\text { Helmholtz Theorem } & 12\end{array}$

2.6 Equivalent Configurations $\quad 20$

2.7 Steady-State Dynamic Problems and Phasor Field $\begin{array}{ll}\text { Representations } & 21\end{array}$

2.8 Continuity Conditions of Fields at a Medium Discontinuity 23 $\begin{array}{ll}\text { References } & 27\end{array}$

3. Problem Definition 28

3.1 Introduction 28

3.2 Field Problem Domains, Source Problem Domains,

3.3 Is the Problem Static, Quasi-Static, or Dynamic? 30

3.4 What Field Is To Be Computed? 31

3.5 Is the Problem Two-Dimensional or Three-Dimensional? 31

$\begin{array}{ll}3.6 \text { The Medium } & 31\end{array}$

3.7 Boundary Conditions and Uniqueness of Solutions 33

$\begin{array}{ll}\text { References } & 35\end{array}$

4. Linear Spaces in Field Computations 36

$\begin{array}{ll}4.1 \text { Introduction } & 36\end{array}$

4.2 Basis Functions $\quad 37$

4.3 Shape Functions $\quad 42$ 
4.4 Finite Elements and Shape Functions of Global Coordinates in Two-Dimensional Problem Domains

4.5 Isoparametric Shape Functions in Two Dimensions

4.6 Finite Elements and Shape Functions of Global Coordinates in Three-Dimensional Problem Domains

References

5. Projection Methods in Field Computations 60

$\begin{array}{ll}5.1 \text { Introduction } & 60\end{array}$

5.2 Special Spaces in Field Computations 62

5.3 Operators in Field Calculations $\quad 65$

5.4 Approaches Used in Obtaining Approximate Solutions to Field Problems

5.5 Finite Element Method for Interior Problems 74

5.6 Integral Equation Method $\quad 78$

$\begin{array}{ll}5.7 \text { Projection Methods } & 81\end{array}$

5.8 Orthogonal Projection Methods $\quad 86$

$\begin{array}{ll}\text { References } & 90\end{array}$

6. Finite Element Method for Interior Problems 91

$\begin{array}{ll}6.1 \text { Introduction } & 91\end{array}$

6.2 Formulation of Finite Element Method for Interior Problems 92

6.3 Computation of Linear System for Finite Element Method 98

6.4 Sample Problem $\quad 105$

$\begin{array}{ll}\text { References } & 110\end{array}$

7. Finite Element Method for Exterior Problems 111

$\begin{array}{ll}7.1 \text { Introduction } & 111\end{array}$

$\begin{array}{ll}\text { 7.2 McDonald-Wexler Algorithm } & 111\end{array}$

$\begin{array}{ll}7.3 \text { Silvester et al. Algorithm } & 120\end{array}$

7.4 Mapping Algorithms 133

References 142

8. Automatic and Adaptive Mesh Generation 143

8.1 Introduction 143

8.2 Preliminary Mesh Generation 146

8.3 Delaunay Tesselation 147

8.4 An Algorithm for Local and Global Error Estimation 149

8.5 Mesh Refinement Algorithm 150

References 151

9. Integral Equation Method 152

9.1 Introduction 152 
9.2 Linear and Uniform Media in Continuity Subdomains

9.3 Saturable, Nonlinear, and Nonuniform Media in Continuity Subdomains

9.4 Numerical Solution of Integral Equations-General Approach

9.5 Finite Elements and Basis Functions Used in the Integral Equation Method

9.6 Integral Equation Numerical Solution by the Collocation Method

9.7 Integral Equation Numerical Solution by the Galerkin Method

9.8 Numerical Integration

9.9 Sample Problem

References

10. Static Magnetic Problem

10.1 Introduction

10.2 Interior Static Field Problems

10.3 Exterior Static Problems Approximated by Interior Problems

10.4 Exterior Magnetic Field Static Problem

10.5 Static Magnetic Field in a Saturable Medium References

\section{Eddy Current Problem}

11.1 Introduction

11.2 Commonly Used Basic Formulations for the Eddy Current Problem

11.3 Two-Dimensional Eddy Current Problem

11.4 Three-Dimensional Steady-State Eddy Current Problem 201

11.5 Transient Eddy Current Problem

Glossary

Appendix A Derivation of the Helmholtz Theorem

Appendix B Properties of the Magnetic Vector Potential, A

Appendix C Proof Regarding Split of Quadrangle into Two Triangles

Appendix D Derivation of Formulations Used in the Cendes-Shenton Adaptive Mesh Algorithm

Appendix E Integral Expressions for Scalar Potential from Green's Theorem 


\section{PREFACE}

Since the first edition of this book was published in 1987, there have been several important changes in the state of numerical field computation, as discussed in the Introduction. These changes have motivated the publication of this second edition.

As with the first edition, the objective of this second edition is to give the newcomer to field computation the information needed to perform practical field computations. Again, clarity of presentation is given greater emphasis than a high degree of sophistication or the state of the art. And again, the basic concepts of field computation are presented as well as the commonly used algorithms.

Several persons have provided much valuable information for this second edition. I wish to thank Professor Giorgio Molinari of the University of Genoa, Italy for advice regarding adaptive mesh generation; Dr. C. R. E. Emson of Vector Fields, Ltd., England and Dr. John Brauer of McNeal-Schwendler Corp. for their advice on transient eddy current computation; and Dr. Zoltan Cendes of Ansoft Corp. for information about their adaptive mesh generator.

Again, I would like to acknowledge the support for this second edition by my wife, Candace. Again, I could not have written this book without her support. 


\section{PREFACE TO THE FIRST EDITION}

For well over a decade, the numerical approach to field computation has been gaining progressively greater importance. Analytical methods of field computation are, at best, unable to accommodate the very wide variety of configurations in which fields must be computed. On the other hand, numerical methods can accommodate many practical configurations that analytical methods cannot. With the advent of high-speed digital computers, numerical field computations have finally become practical.

However, in order to implement numerical methods of field computation, we need algorithms, numerical methods, and mathematical tools that are largely quite different from those that have been traditionally used with analytical methods. Many of these algorithms have, in fact, been presented in the large number of papers that have been published on this subject in the last two decades. And to some of those who are already experienced in the art of numerical field computations, these papers, in addition to their own original work, are enough to give them the knowledge that they need to perform practical numerical field computations.

But newcomers to the art of numerical field computations need a more orderly presentation of the necessary background information than is available to them in these papers. The objective of this book is precisely to provide this orderly presentation of the necessary information. Clarity of presentation is given greater emphasis than a high degree of sophistication or being up to the state of the art. It is my hope that those who read this book will then be able to read and understand the recent papers and therby achieve the level of sophistication to which they aspire.

Specifically, this book has the objective of presenting information in two different categories:

1. Certain algorithms that have been used successfully for computation of fields.

2. Background information that will help readers to develop their own algorithms.

Putting it another way, the intent of this book is to help readers develop or select the algorithms to be used in these field computations. These algorithms 
can then be used in computer programs, and these computer programs can be run in high-speed digital computers to compute the fields.

To build these computer programs, the programmer requires knowledge in a number of other areas beyond the algorithms that are discussed in this book. These areas include computer programming, methods for numerical solution of systems of equations, methods for automatic deployment of node points, and construction of finite elements and computer graphics (to display the computed fields). These important topics are discussed in technical journals as well as in other books.

Any numerical field computation is built upon logic that is necessarily an intimate mixture of mathematics, numerical analysis, and electromagnetic theory. This book is just such a mixture. It is assumed that the reader has some prior knowledge of electromagnetic theory, vector analysis, and linear algebra.

This book covers static and quasi-static field problems in media that generally vary in permittivity, permeability, and conductivity. It does not cover dynamic problems, such as antennas, scatterers, radiation in free space, and waveguide modes. These items are topics for books in themselves.

Here we consider only problems in which both the observer of the fields and the medium throughout the entire domain are fixed with respect to a single frame of reference. That is, we do not consider problems in which a portion of the medium moves with respect to the remainder of the medium (and the field is computed in both)-for example, problems in which the field is to be computed in both the moving rotor and stator of an electric motor or generator. Such problems are an important study in themselves.

An objective of this book has been to use standard nomenclature as much as possible. All of the equations related to electric and magnetic fields are based upon the use of the SI system of units. The symbols used are, for the most part, taken from the international recommendations for quantities, units, and their symbols, as published by the United States of America Standards Institute.

I am deeply indebted to a number of people for their contributions to this book. I appreciate my discussion with Mr. Charles Trowbridge, of Rutherford Laboratories, United Kingdom, regarding my approach to Chapter 5. Dr. Alvin Wexler of the University of Manitoba made valuable suggestions related to my presentation of certain of his material in Chapter 7. In addition, Mr. Trowbridge and Dr. Wexler provided thorough technical reviews of my completed manuscript. Dr. Dennis Lindholm of Ampex Corporation reviewed the majority of the manuscript and made many valuable suggestions. Mrs. Bernie Jones of Palo Alto, California, did an excellent job of typing a large portion of the manuscript.

Finally, and most important, I wish to acknowledge my wife, Candace. She has gently tolerated my work on this book over four annual vacations and 
countless weekends. Without her unfailing support and encouragement I could not have written it.

If the reader wishes to communicate with the author regarding this book, he can send the communication to the publisher, who will then forward it to the author.

Charles W. Steele 